\title{
miR-125b Disrupts Mitochondrial Dynamics via Targeting Mitofusin 1 in Cisplatin-Induced Acute Kidney Injury
}

\author{
Yue Zhao Yue Lang Mingchao Zhang Shaoshan Liang Xiaodong Zhu \\ Zhihong Liu
}

National Clinical Research Center of Kidney Diseases, Jinling Hospital, Medical School of Nanjing University, Nanjing, China

\section{Keywords}

miR-125b · Acute kidney injury · Cisplatin · Mitochondrial fragmentation

\begin{abstract}
Background: Mitochondria are dynamic organelles whose structure are maintained by continuous fusion and fission. During acute kidney injury (AKI) progression, mitochondrial fission in renal tubular cells was elevated, characterized by mitochondrial fragmentation. It is tightly associated with mitochondrial dysfunction, which has been proven as a critical mechanism responsible for AKI. However, the initiating factor for the disruption of mitochondrial dynamics in AKI was not well understood. Objectives: To explore the molecular mechanisms of mitochondrial disorders and kidney damage. Methods: We established cisplatin-induced AKI model in C57BL/6 mice and proximal tubular cells, and detected the expression of miR-125b by qPCR. Then we delivered miR$125 \mathrm{~b}$ antagomir after cisplatin treatment in mice via hydrodynamic-based gene transfer technique. Subsequently, we performed luciferase reporter and immunoblotting assays to prove miR-125b could directly modulate mitofusin1 (MFN1) expression. We also tested the role of miR-125b in mitochondrial and renal injury through immunofluorescent staining, qPCR, and immunoblotting assays.
\end{abstract}

Results: miR-125b levels were induced in cisplatin-challenged mice and cultured tubular cells. Anti-miR-125b could effectively alleviate cisplatin-induced mitochondrial fragmentation and kidney injury both in vitro and in vivo. Furthermore, miR-125b could directly regulate MFN1, which is a key regulator of mitochondrial fusion. Our study indicated that miR-125b is upregulated during cisplatin-induced AKI. Inhibition of miR-125b may suppress mitochondrial and renal damage through upregulating MFN1. This study suggests that miR-125b could be a potential therapeutic target in AKI.

(c) 2021 The Author(s)

Published by S. Karger AG, Basel

\section{Introduction}

Mitochondria are dynamic cellular organelles whose structure is maintained by continuous fusion and fission [1]. Mitochondria are generally elongated and filamentous in physiological conditions, and become fragmented in response to stress stimulation $[2,3]$. The abnormal mitochondrial structure is tightly associated with mitochondrial dysfunction and disease pathology [4]. The most crucial function of mitochondria is the production of

Yue Zhao and Yue Lang contributed to this work equally. karger@karger.com www.karger.com/kdd

Karger $\stackrel{\text { ' }}{5}$

BOPEN ACCESS
(C) 2021 The Author(s)

Published by S. Karger AG, Basel

This is an Open Access article licensed under the Creative Commons Attribution-NonCommercial-4.0 International License (CC BY-NC) (http://www.karger.com/Services/OpenAccessLicense), applicable to the online version of the article only. Usage and distribution for commercial purposes requires written permission.
Correspondence to:

Zhihong Liu, liuzhihong@nju.edu.cn 
ATP through oxidative phosphorylation [5]. The kidney has a high energy demand due to its active reabsorption of large quantities of solutes. However, the proximal tubular cells (PTCs) have very limited capacity for glycolysis. Instead, they are very rich in mitochondria in order to supply energy via oxidative phosphorylation $[5,6]$. Thus, the stability of mitochondria structure and function is critical to the maintenance of normal kidney function.

Mitochondrial dysfunction leads to reduced ATP production and the generation of reactive oxygen species (ROS) which may contribute to the pathological process of acute kidney injury (AKI). AKI is associated with a rapid decline of renal function. It is a worldwide public health problem with increased morbidity and mortality [7]. The causes of AKI mainly include ischemia-reperfusion, sepsis, and various exogenous nephrotoxins [8]. During the development of AKI, mitochondrial fission in tubular cells is elevated, and characterized by mitochondrial fragmentation, which promotes cell apoptosis and renal injury [9]. Recently, more and more studies focus on improving mitochondrial function to prevent PTC injury and restore renal function during AKI. Despite extensive research, the molecular mechanism of mitochondrial abnormalities under the AKI pathological condition remains unclear.

microRNAs are endogenous small, noncoding RNAs of 21-25 nucleotides that play vital roles in gene expression regulation through translational repression and/or mRNA degradation of target genes [10]. In recent years, the relationship between miRNA and mitochondrial damage has received increasing attention. In 2010, Wei et al. [11] established a mouse model with specific ablation of Dicer from renal proximal tubules. These mice were deprived of miRNAs in tubular cells and became resistant to ischemic AKI, demonstrating an important role of miRNAs in AKI [11]. After then, several studies found that lots of miRNAs could participate in the AKI process via regulating mitochondrial morphology and function, such as miR-484 and miR-709 $[12,13]$. In this study, we found that miR-125b was significantly upregulated in response to cisplatin treatment both in vitro and in vivo. miR-125b is a highly conserved miRNA which participates in many physiological and pathological processes $[14,15]$, considering that miR-125b exerted a vital role in mitochondrial metabolism and dynamics regulation in monocyte [16]. We hypothesized that miR-125b could also influence the mitochondrial homeostasis in renal tubular cells via regulating its target genes during AKI.

In this study, with bioinformatic analysis, we predicted that miR-125b could directly target and regulate the pro- fusion molecule mitofusin1 (MFN1). MFN1 is a key regulator of mitochondrial fusion, which is linked to mitochondrial biogenesis and respiratory functions, impacting cell fate and organism homeostasis [17]. MFN1 maintains a certain abundance of expression under the physiological condition and could be downregulated during the stimulation by stress or injury factors. Modulation of MFN1 levels has been proven to prevent mitochondrial fission and cell apoptosis in various cell context [18, 19]. As so, we speculate whether miR-125b promotes cisplatin-induced mitochondrial dysfunction and kidney injury through inhibiting MFN1 levels. With a series of in vivo and in vitro experiments, we confirmed that MFN1 is a direct target of miR-125b. During cisplatin nephrotoxicity, miR-125b is upregulated and then inhibits MFN1 levels, thereby promoting mitochondrial division and tubular injury.

\section{Materials and Methods}

\section{Cell Culture and Treatment}

The human renal PTC (HK2) cell line was obtained from the American Type Culture Collection and cultured in DMEM/F-12 medium (Gibco; Thermo Fisher Scientific) supplemented with $10 \%$ fetal bovine serum, penicillin $(100 \mathrm{U} / \mathrm{mL})$, and streptomycin $(100 \mu \mathrm{g} / \mathrm{mL})$, and maintained at $37^{\circ} \mathrm{C}$ in $5 \% \mathrm{CO}_{2}$ in a humidified incubator. The cells were serum-starved for $12 \mathrm{~h}$ before cisplatin treatment. The cells were treated with $20 \mu \mathrm{M}$ cisplatin for $24 \mathrm{~h}$.

\section{Animal Experiments}

We obtained the male C57BL/6 mice (8-10 weeks) from the Model Animal Research Center of Nanjing University (Nanjing, China). The use of animals in this study was approved by the Institutional Animal Care and Use Committee at Jinling Hospital. Male C57BL/6 mice were used to generate the cisplatin-induced AKI model. Cisplatin $(20 \mathrm{mg} / \mathrm{kg}$ body $\mathrm{wt})$ was given through intraperitoneal injection as described previously [20]. The mice in the control group were injected with normal saline. All the mice were euthanized $72 \mathrm{~h}$ after cisplatin administration. Kidneys and blood were collected after the animals were sacrificed. To reduce miR-125b levels in the mice kidney, miR-125b antagomir (GenePharma, Shanghai, China) was administered to the mice using a previously described hydrodynamic-based gene transfer technique. Briefly, $40 \mu \mathrm{g}$ of antagomir in approximately $2.6 \mathrm{~mL}$ TransIT-EE Hydrodynamic Delivery Solution (Mirus) was injected into a mice via tail vein in 5-7 s.

\section{Transmission Electron Microscopy and Mitochondrial} Fragmentation

Renal cortex tissues were fixed in $3.75 \%$ glutaraldehyde and post-fixed in $1 \%$ osmium tetroxide. After dehydration in ethanol, the specimens were embedded in epon. Ultrathin sections in $70 \mathrm{~nm}$ were stained and examined with Hitachi 7500 transmission electron microscope (Hitachi, Tokyo, Japan). Mitochondria whose length $>2 \mu \mathrm{m}$ were considered filamentous, and those with length 

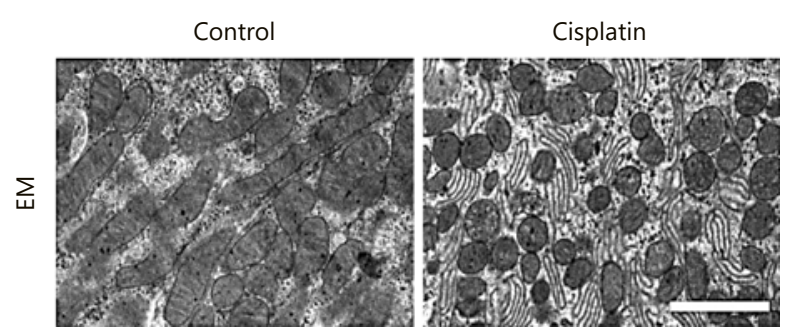

a
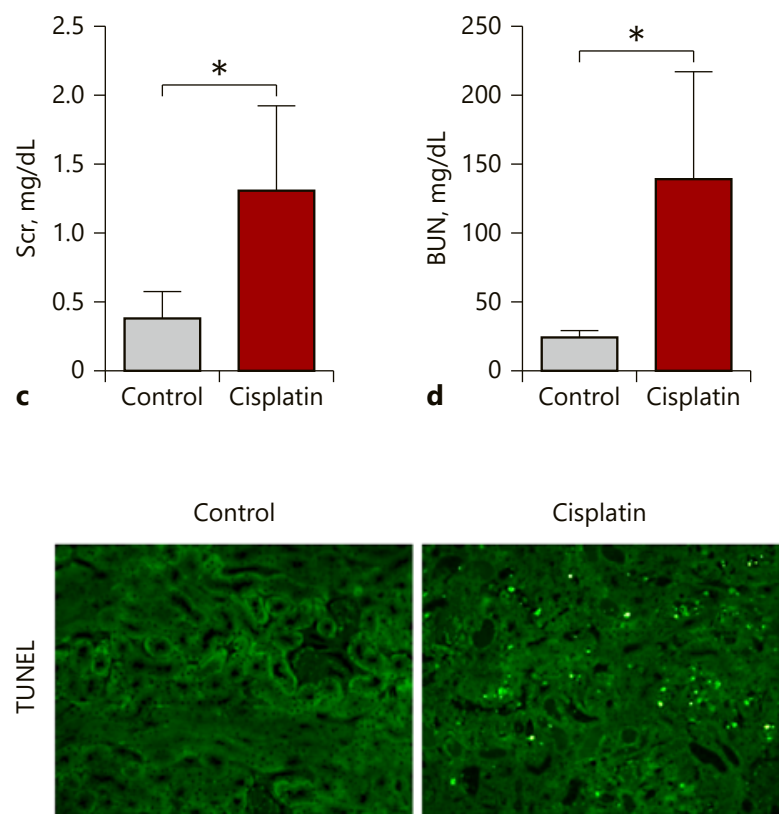

$\mathbf{f}$
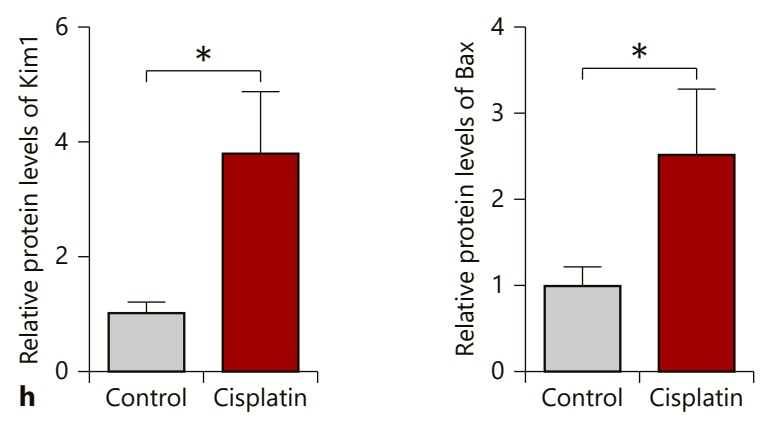

Fig. 1. Cisplatin-induced mitochondrial dysregulation and $A K I$ in mice. C57BL/6 mice (male, 8-10 weeks) were given cisplatin (20 $\mathrm{mg} / \mathrm{kg}$ ) or normal saline (control) i.p. on day 0 and sacrificed on day 3. a Representative micrographs of electron microscopy of kidney PTCs from normal saline and cisplatin-treated mice (scale bar $=2 \mu \mathrm{m}$ ). Quantification of the fragmented mitochondria is shown on the right. $\mathbf{b}$ qPCR analysis of mtDNA copy number. $\mathbf{c}$ Scr levels in each group. d Serum BUN levels in each group. e Representative images of renal PAS staining (scale bar $=50 \mu \mathrm{m}$ ). f Repre-
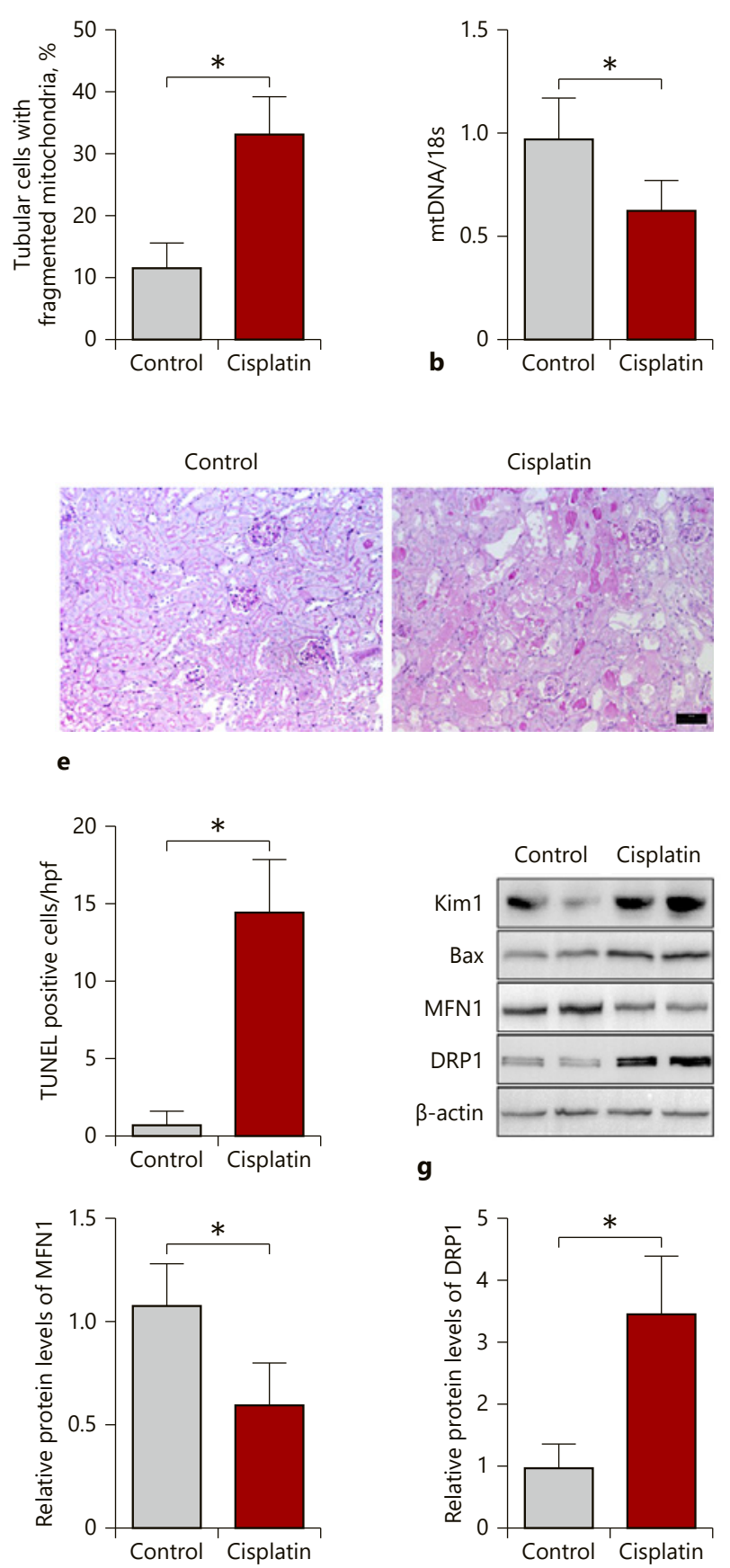

sentative images of TUNEL staining. Quantification of TUNELpositive cells in the kidney (scale bar $=40 \mu \mathrm{m}$ ). $\mathbf{g}$ Western blotting analysis of Kim-1, Bax, MFN1, and DRP1 expressions in cisplatin or normal saline-treated mice. $\mathbf{h}$ Quantification of g. Student's $t$-test was used, ${ }^{*} p<0.05$ versus control. Data are shown as mean \pm SD ( $n=5-6$ in each group). AKI, acute kidney injury; i.p., intraperitoneal injection; PTCs, proximal tubular cells; qPCR, quantitative PCR; Scr, serum creatinine; PAS, periodic acid-Schiff; MFN1, mitofusin1. 
$<1 \mu \mathrm{m}$ and spherical configuration were defined as fragmented. The percentage of tubular cells with $<1 \%$ long filamentous mitochondria was quantified, indicating the degree of mitochondrial fragmentation according to the previous literature [2].

\section{mtDNA Copy Number}

Renal cortex was extracted using a Tissue DNA Kit (OMEGA) according to the manufacturer's instructions. Quantitative PCR was performed to detect the copy number of mtDNA. Relative mtDNA copy numbers were normalized to the nuclear $18 \mathrm{~S}$ rRNA. The primer sequences were listed as follows: mtDNA: ATCCTCCCAGGATTTGGAAT, ACCGGTAGGAATTGCGATAA; 18s rRNA: TTCGGAACTGAGGCCATGATT, TTTCGCTCTGGTCCGTCTTG.

\section{Serum Biochemistry}

We centrifuged the whole blood of mice at $1,000 \mathrm{~g}$ for $15 \mathrm{~min}$ to separate serum. The biochemistry analysis of serum creatinine (Scr) and urea nitrogen (BUN) from mice were measured by the fully automatic bio analysis machine (Rayto, China).

\section{Periodic Acid-Schiff (PAS) Staining}

Kidney tissues were embedded in paraffin, and 3- $\mu$ m sections were prepared and stained with the PAS reagent. The images of each section were pictured with the Leica microscope (DM5000B).

\section{TUNEL Assay}

TUNEL assays were performed to detect cell apoptosis with the In Situ Cell Death Detection Kit, POD (Roche) following the manufacturer's instructions.

\section{Western Blotting}

The kidney cortex and cells were lysed in radio immunoprecipitation assay buffer containing protease inhibitor cocktail (Roche) and phosphatase inhibitor. The primary antibodies used were as follows: Kim1 (Absin), Bax (Proteintech), MFN1 (Abcam, Cambridge, MA, USA), DRP1 (Santa Cruz, CA, USA), and b-actin (Affinity Biosciences). Quantification was performed by measuring the intensity of the bands with Image-Pro plus 6.0 software (Media Cybernetics, Rockville, MD, USA).

\section{RNA Extraction and $q R T-P C R$ Assays}

Total RNA was extracted from cells and kidney tissue with TRIzol Reagent (Invitrogen) according to the manufacturer's instructions. TaqMan miRNA Assay Probes (Applied Biosystems, Foster City, CA, USA) were used to quantify the miR-125b levels in the RNA samples. Real-time PCR was performed using a TaqMan PCR kit on the 7900HT Sequence Detection System (Applied Biosystems). We normalized the miRNA expressions to U6 snRNA.

\section{Luciferase Reporter Assay}

The 3'-UTR of MFN1 was obtained by PCR using human genomic DNA and inserted downstream of the pmirGLO-promoter (Promega). We mutated the sequences in MFN1 3'UTR that interact with the miR-125b seed sequence. For the luciferase reporter assay, we transfected the constructs combined with miR-125b mimic or nc mimic into 293T using GP-transfect-Mate (GenePharma). $24 \mathrm{~h}$ later, cell lysates were prepared and luciferase activities were measured using the Dual-Luciferase Report Assay System (Promega).
Assessment of Mitochondrial Fragmentation in vitro

Cells were seeded onto coverslips. After different treatments, cells were incubated with MitoTracker (Life Technologies) and then fixed with paraformaldehyde. Then the slides were mounted with Antifade mounting medium for further examination using confocal fluorescent microscopy. 150-200 cells from 10 randomly selected fields were examined for each slide to quantify the percentage of cells with mitochondrial fragmentation.

\section{Mitochondrial ROS}

Mitochondrial ROS levels were measured using mitoSOX red mitochondrial superoxide indicator (Invitrogen) as previously described [21].

\section{Statistical Analysis}

The data are presented as the mean $\pm \mathrm{SD}$. Analyses were performed with GraphPad Prism 8 software (GraphPad Software Inc., La Jolla, CA, USA). The normality test of the data was performed by the Shapiro-Wilk test. Differences between groups were compared using the Student's $t$-test or ANOVA method, and post hoc analyses were analyzed using the Bonferroni correction or Dunnett correction. $p<0.05$ was considered statistically significant.

\section{Results}

\section{Cisplatin Treatment Caused Mitochondrial}

Fragmentation and Kidney Injury in Mice

In order to generate the cisplatin-induced AKI model, male C57BL/6 mice were intraperitoneally injected cisplatin at a dose of $20 \mathrm{mg} / \mathrm{kg}$. Mice were sacrificed on day 3 , and kidney tissue were collected for subsequent analysis. We observed the morphology of mitochondria in mouse kidneys cortices with transmission electron microscopy. EM micropraphs showed that compared with the normal saline group, many PTCs fragmented their mitochondria into small, round suborganelles. Quantitative morphometric analysis confirmed that the PTCs with fragmented mitochondria were increased in cisplatintreated mice (Fig. 1a). At the molecular level, cisplatin insult affected the level of the main proteins, which regulates mitochondria fission and fusion. Downregulation of MFN1 and upregulation of DRP1 were detected by Western blotting (Fig. 1g, h). Furthermore, mtDNA copy number was declined in response to cisplatin treatment (Fig. 1b), suggesting less of mitochondria. After cisplatin injection, the levels of BUN and SCr were increased on day 3 (Fig. 1c, d). In addition, PAS staining of the renal sections showed that cisplatin-injected mice displayed kidney injury phenotype, such as loss of the brush border, tubular cell loss, and cast formation (Fig. 1e). Western blotting showed that Kim 1 levels were increased after cisplatin injection, which indicated cisplatin could induce 


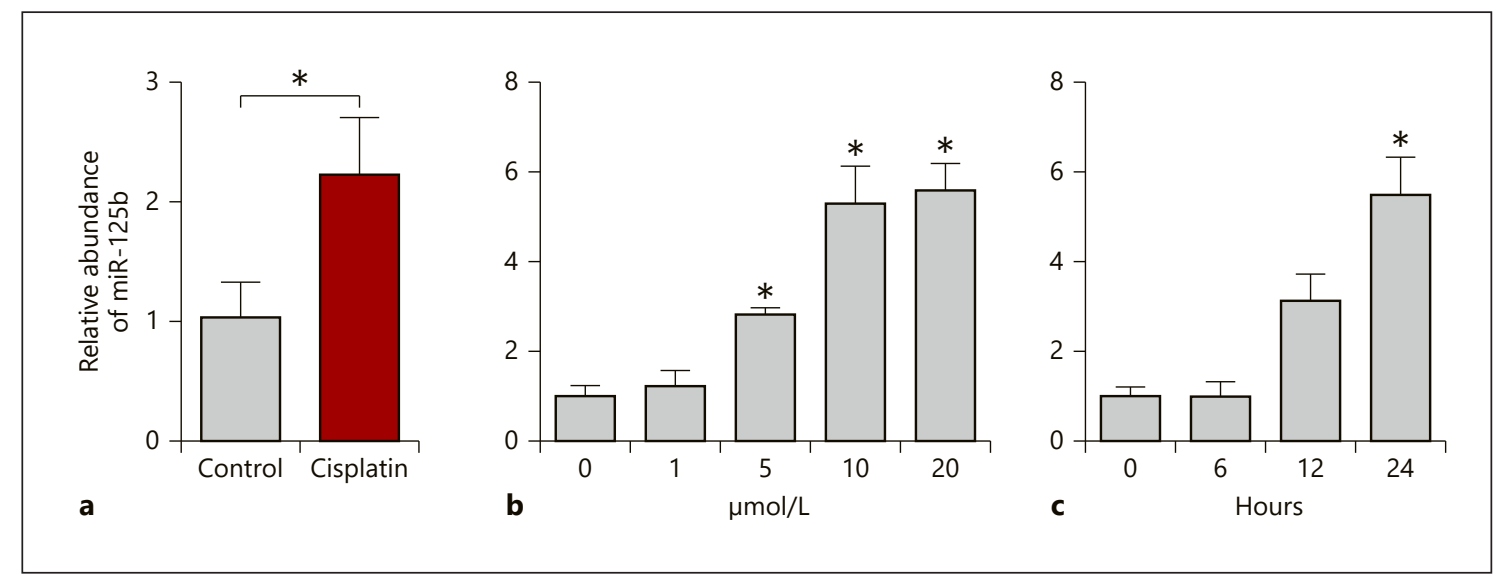

Fig. 2. Cisplatin treatment upregulated miR-125b levels both in vivo and in vitro. a qRT-PCR analysis of miR-125b in cisplatintreated mouse kidney on day3. Student's $t$-test was used, ${ }^{*} p<0.05$ versus control (normal saline). Data are shown as mean $\pm \mathrm{SD}(n=$ $5-6$ per group). $\mathbf{b}$ qPCR analysis of miR-125b in renal tubular cells exposed to cisplatin insult at various dosages $(0-20 \mu \mathrm{M})$. One-way ANOVA was used, and post hoc analyses were analyzed using the Dunnett T correction. ${ }^{*} p<0.05$ versus $0 \mu \mathrm{M}$. Data are presented as

renal damage (Fig. 1g, h). We next assessed apoptotic cells in kidneys using TUNEL staining. As shown in Figure 1f, TUNEL-positive cells were increased in the cisplatin group. Accordingly, cisplatin treated also induced the expression of the proapoptotic protein Bax (Fig. 1g, h). Taken together, these data demonstrated that cisplatin could induce AKI and mitochondrial damage in vivo.

\section{Cisplatin Upregulated miR-125b Levels Both in vivo} and in vitro

In our previous work, we found that plasma miR-125b levels were upregulated in nephrotic syndrome patients relative to healthy controls, which indicated the critical role of miR-125b in nephropathy [22], considering the vital role of miR-125b in mitochondrial metabolism and dynamics [23]. We questioned whether miR-125b was involved in cisplatin-induced nephropathy. First of all, qPCR was performed to evaluate the miR-125b expression in the cisplatin-induced AKI model. The results showed the upregulation of miR-125b in the renal cortex of cisplatin injection (Fig. 2a). Next, cultured tubular epithelial cells were given different doses of cisplatin $(0,1$, $5,10$, and $20 \mu \mathrm{M})$ for $24 \mathrm{~h}$, or treated with $20 \mu \mathrm{M}$ cisplatin in various time points ( $0 \mathrm{~h}, 6 \mathrm{~h}, 12 \mathrm{~h}$, and $24 \mathrm{~h}$ ), and then miR-125b expressions were detected (Fig. 2b, c). Our results found that cisplatin could induce overexpression of miR-125b in a dose- and time-dependent manner.

miR-125b Downregulates Mitofusin 1 to Induce Kidney Damage the mean \pm SD of 3 independent experiments. c qPCR analysis of miR-125b in cisplatin-exposed HK2 for different time points $(0,6$, 12 , and $24 \mathrm{~h}$ ). Differences between the groups at time point were analyzed by repeated measures one-way ANOVA, and post hoc analyses were analyzed using the Dunnett T correction. ${ }^{*} p<0.05$ versus $0 \mathrm{~h}$. Data are presented as the mean \pm SD of 3 independent experiments. qPCR, quantitative PCR.

\section{Anti-miR-125b Alleviated Kidney Injury and Mitochondrial Dysfunction in Cisplatin-Induced AKI} Mice

To determine the role of miR-125b in vivo, we tested the effect of miR-125b inhibition on the cisplatin-induced AKI model. miR-125b antagomir or nc was administered to mice using the TransIT in vivo gene delivery system (Mirus) [24] on the second day after cisplatin injection (Fig. 3a). As expected, cisplatin-induced mir-125b upregulation was abrogated after anti-mir-125b transfer (Fig. 3b). To evaluate the influence of anti-miR-125b on mitochondrial morphology, we observed the ultrastructure of mitochondria using transmission electron microscope. The increased mitochondrial division in cisplatin-induced AKI mice was attenuated after miR-125b antagomir administration (Fig. 3c). In line with the alterations in mitochondrial morphology, the elevated levels of DRP1 in cisplatin-treated mice were decreased in the anti-miR-125b-administrated group. In addition, cisplatin-induced MFN1 decrease was rescued through miR-125b inhibition (Fig. 3j, k). Moreover, qPCR analysis showed that cisplatin-downregulated mtDNA copy number was restored by miR-125b antagomir (Fig. 3d). Notably, in addition to improve mitochondrial fragmentation, inhibition of miR-125b also relieved cisplatin-induced damage to kidney function. As shown in Figure $3 e$ and $\mathrm{f}$, both Scr and BUN increased by cisplatin treatment were reduced after miR-125b an- 

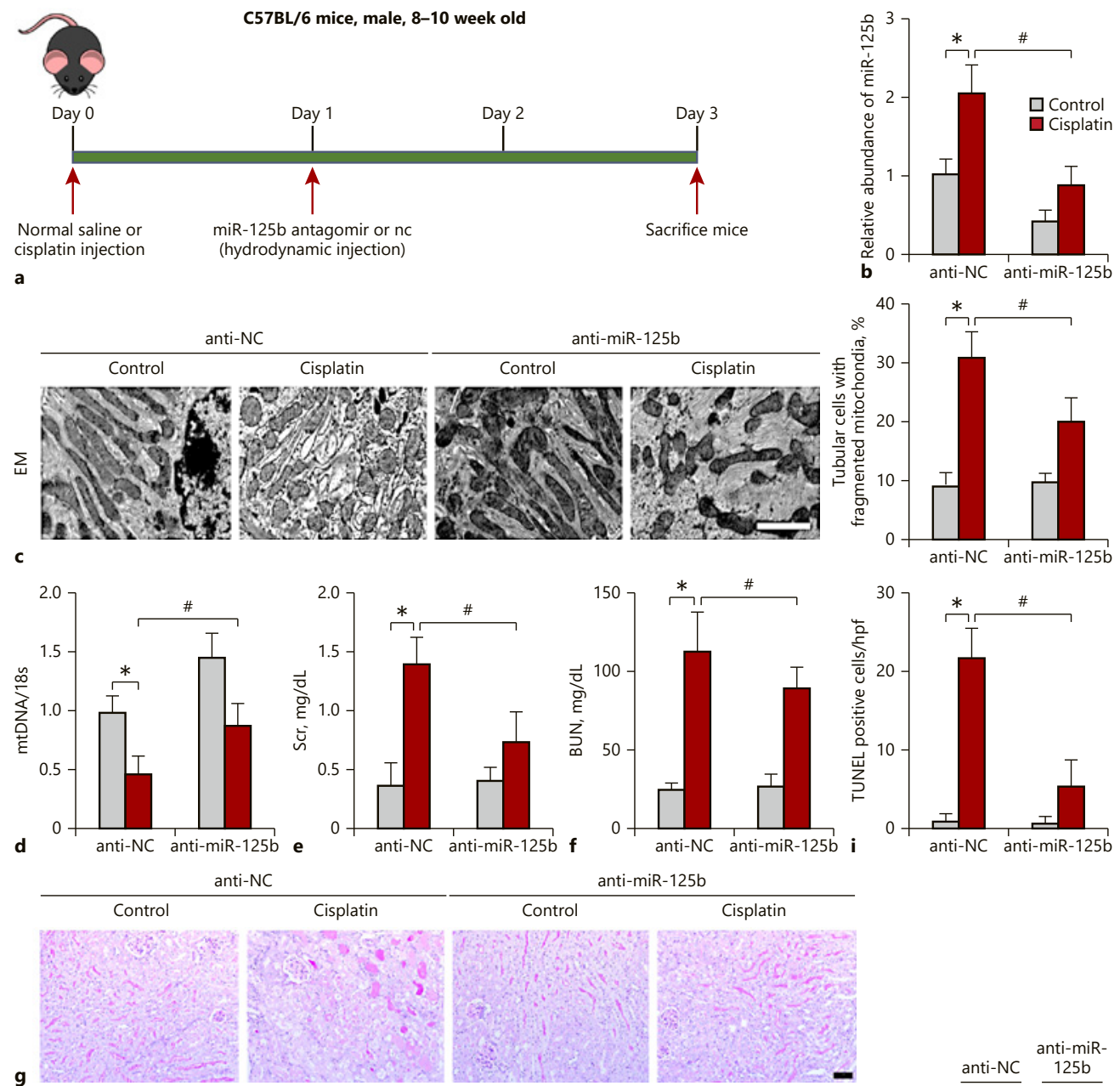

anti-NC

anti-miR-125b
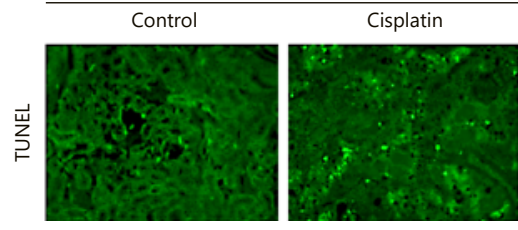

Control
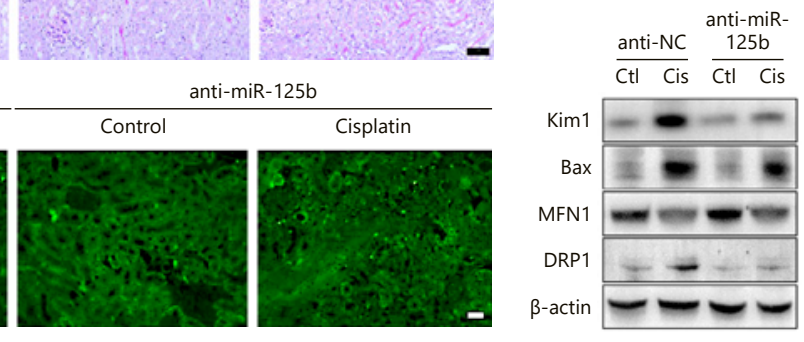

j
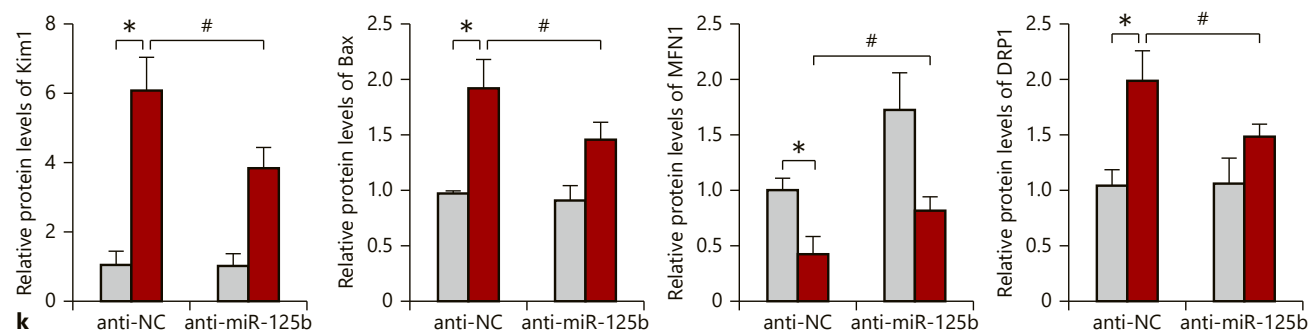

3

(For legend see next page.) 
tagomir delivery. Consistently, miR-125b antagomir ameliorated the histological damage in renal tubules (Fig. 3g) as well as Kim1 levels (Fig. 3j, k). Cisplatin-induced apoptosis was also decreased by miR-125b inhibition, as shown by the TUNEL assay (Fig. 3h, i) and Bax expression (Fig. 3j, k). Collectively, these results supported that anti-miR$125 \mathrm{~b}$ could effectively alleviate mitochondrial fragmentation and tubular injury in cisplatin-induced nephritis.

\section{miR-125b Directly Modulates MFN1 Expression}

To explore the mechanism underlying the effects of miR-125b on cisplatin-induced kidney injury, we searched the putative targets for miR-125b through TargetScan, the miRNA target analyzing database, and predicted MFN1as a miR-125b target (Fig. 4a). MFN1 plays a vital role in mitochondrial fusion, and we also observed MFN1 down-regulation in kidneys of cisplatin-treated AKI mice. Therefore, we wondered whether miR-125b participates in cisplatin-induced AKI through directly inhibiting MFN1. To assess whether miR-125b directly targets the $3^{\prime}$-UTR of MFN1, we performed luciferase reporter assays using reporters carrying either the wild-type (WT) or mutant MFN1 3'UTR (Fig. 4a). WT or mutant reporters and miR-125b mimic or nc mimic were cotransfected in $293 \mathrm{~T}$ cells, and then luciferase activity was detected. The results showed that the activity of WT luciferase was significantly decreased by miR-125b-mimic compared with nc-mimic. However, mutation of the predicted seed region completely abolished the effects of miR-125b-mimic on reporter gene expression (Fig. 4b).

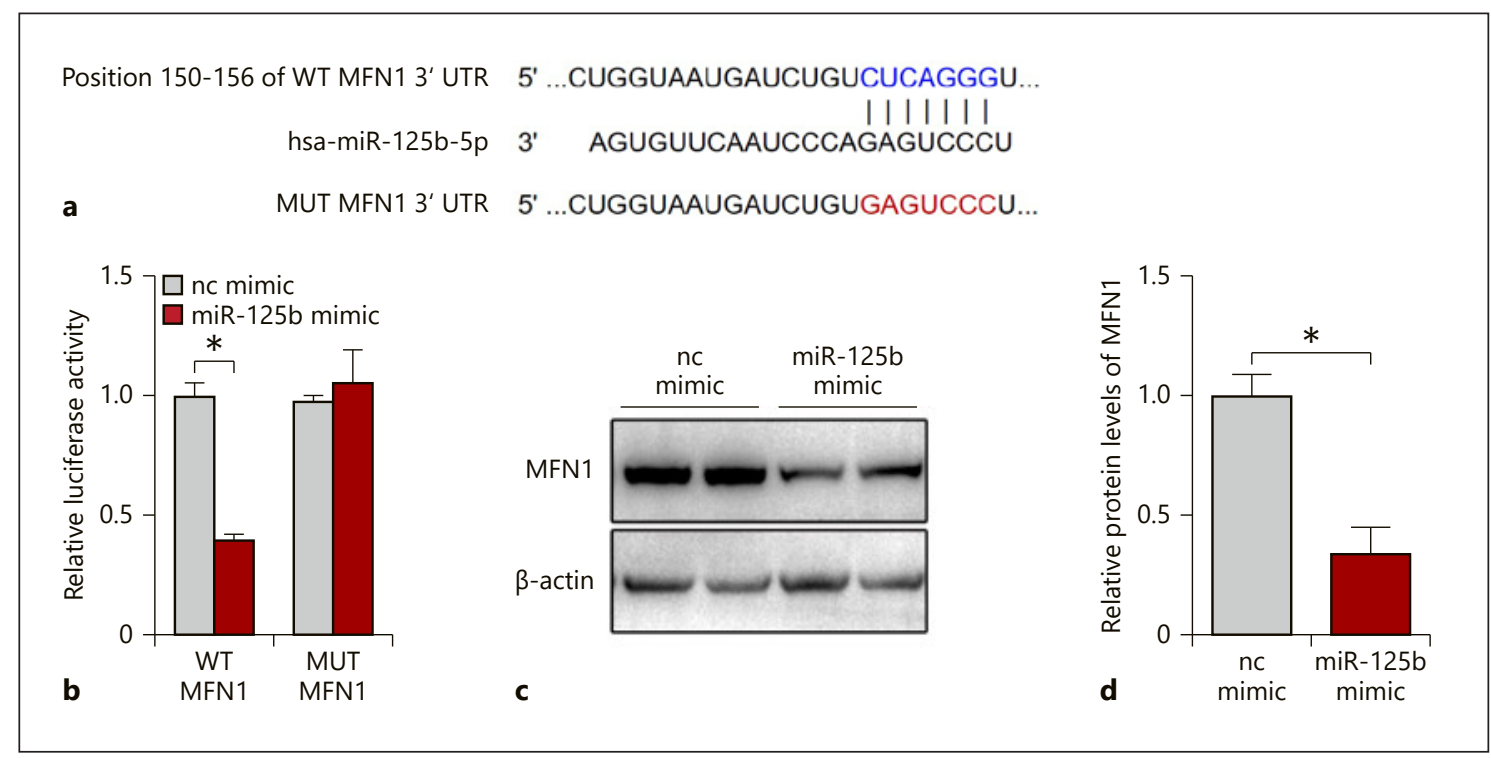

Fig. 4. MFN1 is a direct target of miR-125b. a The predicted miR$125 \mathrm{~b}$ binding site in the MFN1 $3^{\prime}$ UTR (Targetscan) and the mutations made in the seed-recognizing sites to create a MUT MFN1 $3^{\prime}$-UTR for the luciferase reporter construction. $\mathbf{b}$ The luciferase assay. Two-way ANOVA was used, ${ }^{*} p<0.05$ versus nc mimic.

Fig. 3. miR-125b antagomir attenuates cisplatin-induced mitochondrial dysfunction and kidney injury. a Schematic diagram of the experimental design. Mice were given cisplatin or normal saline through i.p. on day 0 . On the second day (day 1), we delivered the miR-125b antagomir and control vector (nc) through tail vein of mice via a previously described hydrodynamic-based genetransfer technique. All the mice were sacrificed on day 3. b qPCR showing that miR-125b antagomir inhibited miR-125b expression effectively. c Representative micrographs of electron microscopy. Quantification of the fragmented mitochondria is shown on the right (scale bar $=2 \mu \mathrm{m}$ ). $\mathbf{d}$ qPCR analysis of mtDNA copy c Immunoblotting of the MFN1 expression in nc or miR-125b mimic transfected tubular cells. d Quantification of C, Student's $t$-test was used, ${ }^{*} p<0.05$ versus nc mimic. All data are presented as the mean \pm SD of 3 independent experiments. MFN1, mitofusin1; MUT, mutant. number. e Scr levels in each group. f Serum BUN levels in each group. g Representative images of PAS staining in different groups (scale bar $=50 \mu \mathrm{m})$. $\mathbf{h}$ Representative images of TUNEL staining (scale bar $=40 \mu \mathrm{m}$ ). i Quantification of TUNEL-positive cells in the kidney. $\mathbf{j}$ Immunoblotting analysis of the expression of Kim-1, Bax, MFN1, and DRP1. k Quantification of J. Two-way ANOVA was used, ${ }^{*} p<0.05$ versus anti-NC + control, ${ }^{*} p<0.05$ versus anti-NC + cisplatin. Data are shown as mean $\pm \mathrm{SD}(n=6-7$ per group). i.p., intraperitoneal injection; qPCR, quantitative PCR; Scr, serum creatinine; PAS, periodic acid-Schiff; MFN1, mitofusin 1 . 


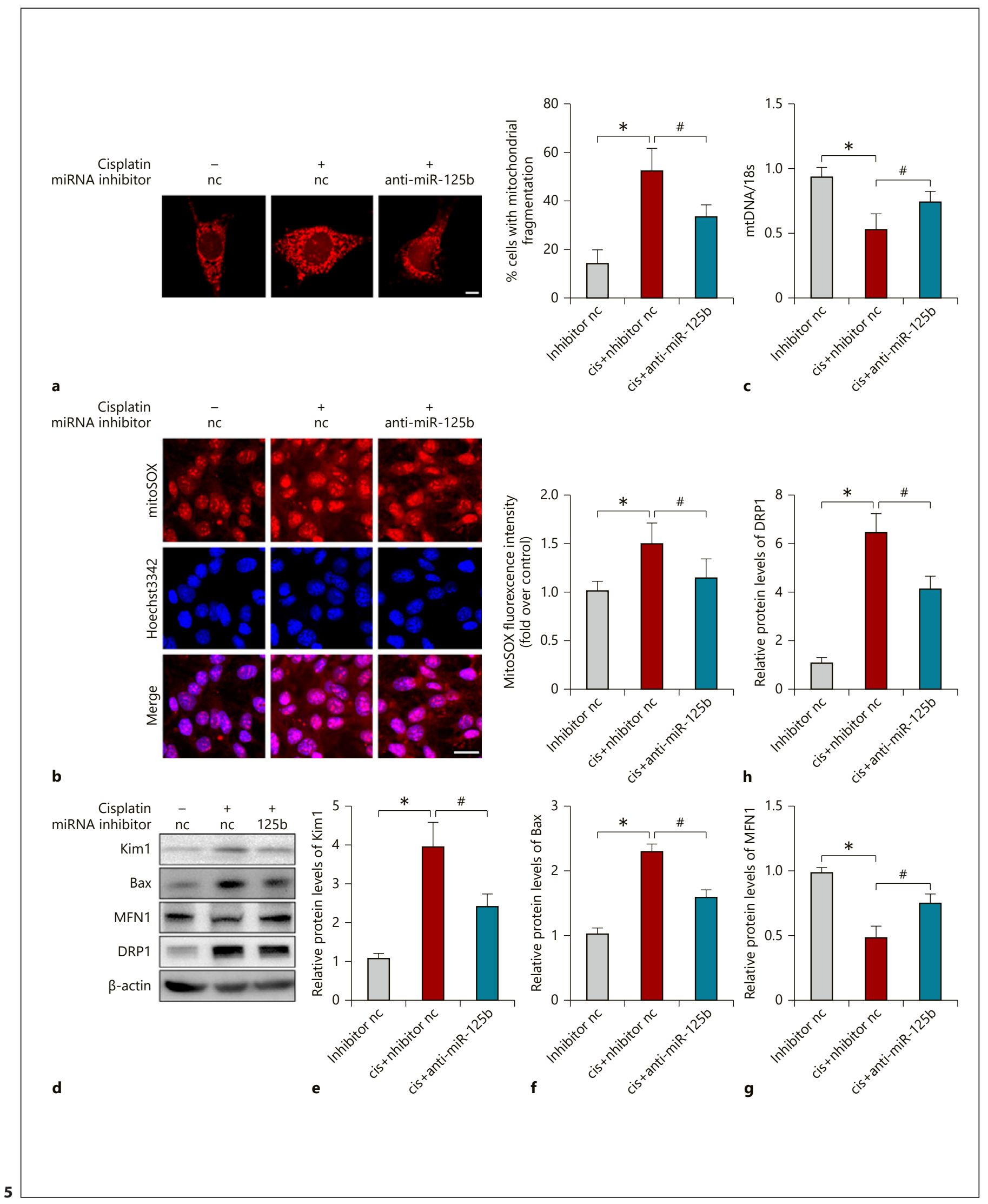

(For legend see next page.) 
In addition, Western blotting showed that the expression of MFN1 was decreased after miR-125b mimic transfection (Fig. 4c, d). All these results indicated that miR-125b could directly target the $3^{\prime}$-UTR of MFN1.

\section{The Protective Effect of Anti-miR-125b in Cisplatin-}

Treated Renal Tubular Cells

To further verify the renal protection of anti-miR$125 \mathrm{~b}$, we compared the effects of miR-125b inhibitor or nc inhibitor transfected cells combined with cisplatin treatment. The MitoTracker assay showed that miR-125b inhibitor could suppress mitochondrial fragmentation during cisplatin treatment, verifying the protective effect of anti-miR-125b (Fig. 5a). Consistently, as shown in Fig. 5b, c, the increased mitochondrial ROS production and the decreased mtDNA copy number induced by cisplatin were reversed after miR-125b inhibition. Furthermore, cisplatin-induced decrease of MFN1 was reversed by anti-miR-125b (Fig. 5d, g). Cisplatin-induced upregulation of DRP1 was downregulated after miR-125b inhibitor transfection (Fig. 5d, h). We also evaluated the apoptosis and injury in anti-miR-125b-transfected renal tubular cells. As shown in Figure 5d andf, cisplatin-upregulated Kim 1 and Bax levels were inhibited by anti-miR-125b. Our results demonstrated that antimiR-125b exerts its mitochondrial and renal protection role in cisplatin-challenged HK2 cells.

\section{Discussion}

Mitochondria are an highly dynamic organelle that change their shape constantly through fission and fusion, which is defined as mitochondrial dynamics [25]. This balance is broken and progress toward division during the AKI pathological process [9]. And the excess of fission may result in mitochondrial fragmentation, which is tightly coordinated with the functional abnormalities [26]. The disruption of the mitochondrial structure also leads to a series of danger signals such as releases of cytochrome c, a trigger of apoptosis, as well as mtDNA [3].

Fig. 5. The injury effects of cisplatin was attenuated in antimiR125b-transfected renal tubular cells. Renal tubular cells were transfected with inhibitor control (NC) or mir-125b inhibitor combined with cisplatin treatment. The cells were then treated with $20 \mu \mathrm{M}$ cisplatin or left untreated as control. a Representative images of Mitotracker staining and quantification of mitochondrial fragmentation in tubule epithelial cells (scale bar $=10 \mu \mathrm{m}$ ). b Representative MitoSOX staining images (red), and nuclei are
Furthermore, ROS released by damaged mitochondria play a vital role in the oxidative stress process during AKI. It is worth noting that mitochondria dysfunction appear earlier than clinical manifestations of kidney injury in AKI $[27,28]$. All these evidence strongly suggested that mitochondria might be a compelling therapeutic target in AKI treatment.

To further explore the mechanism of mitochondrial injury, we established the AKI animal model via a single dose injection of cisplatin $(20 \mathrm{mg} / \mathrm{kg})$. After 3 days, the mice showed renal pathological changes, renal function decline, and increased mitochondrial division. Besides mitochondrial structure abnormity, the excessive of ROS production as well as the decrease of mtDNA copy number were also evoked by cisplatin in renal tubular cells, which were consistent with other literature [29]. Based on this injury model, we revealed the critical role of miR$125 \mathrm{~b}$ in mitochondrial dynamic and kidney injury regulation in this study. In our previous work, we had found that miR-125b levels were upregulated in plasma of focal segmental glomerulosclerosis (FSGS) patients relative to healthy controls [22]. Another study discovered that miR-125b levels in the kidney cortex were significantly upregulated in different types of kidney diseases by deep sequencing [30]. miR-125b was a multifaceted molecule, which can act as a cancer promoter or a cancer repressor depending on the cell context [31]. It plays crucial roles in various pathological and physiological processes like cell differentiation, proliferation, and apoptosis [32]. Zhang and colleagues [33] identified that miR-125b could promote apoptosis through regulating $\mathrm{Mcl}-1, \mathrm{Bcl}-\mathrm{w}$, and IL-6R in hepatocellular carcinoma. Furthermore, it has been reported that miR-125b has the ability to regulate the mitochondria dynamic and function in monocyte [16]. Recently, growing evidence indicated that miRNA could play an important role in mitochondrial structure and function. It has been reported that mir-668 inhibited MTP18 to preserve mitochondrial dynamics and the viability of renal tubular cells in ischemic AKI [13]. miR499 was proven to be able to prevent cardiomyocyte apoptosis by targeting DRP1 activation and consequent mi- counterstained with hoechst3342 (blue). MitoSOX flurescence intensity was quantified on the right, which indicated mitochondrial ROS levels (scale bar $=20 \mu \mathrm{m}$ ). c qPCR analysis of mtDNA copy number in different groups. $\mathbf{d}$ Immunoblotting analysis of the expression of Kim-1, Bax, MFN1, and DRP1. e-h Quantification of d. One-way ANOVA was used, ${ }^{*} p<0.05$ versus inhibitor $\mathrm{nc}, \# p<$ 0.05 versus cis + inhibitor nc. Data are shown as mean \pm SD. MFN1, mitofusin1; qPCR, quantitative PCR. 
tochondrial division [34]. These reports illustrated that targeting mitochondrial damage has become a potential strategy for disease therapy.

Based on these clues, we speculated whether miR-125b was involved in cisplatin nephritis through mitochondria regulation. Our results showed that miR-125b levels were increased after cisplatin administration both in vitro and in vivo. Furthermore, inhibit miR-125b expression with miR-125b antagomir injection could alleviate the mitochondrial dysfunction as well as kidney injury. These results indicated that miR-125b plays a critical role in cisplatin-induced mitochondrial and renal damage. Considering its short sequence, miR-125b is convenient for administration and therefore has the potential to become an effective target for the AKI treatment.

Next, we further explore the mechanism of miR-125bregulated mitochondrial and renal injury. Through the bioinformatics database searching, we found that miR$125 \mathrm{~b}$ is predicted to directly target and regulate MFN1. MFN1 is a large GTPase that belongs to mitofusin protein family. Mitofusins are key components involved in the mitochondrial fusion, which include MFN1 and MFN2. Lots of evidence illustrated that MFN1 plays a critical role in mitochondrial morphology and cell injury. It has been shown that MFN1 is decreased in response to injury factors and then leads to the mitochondrial membrane permeabilization, which contributes to mitochondrial fission and apoptosis $[18,19,35]$. However, MFN2 has also been reported as a modulator of mitochondrial function through affecting its dynamics $[36,37]$. It is noteworthy that MFN1 regulated mitochondrial docking and fusion more efficiently than MFN2, due to its higher GTPase activity [38]. Besides, MFN1 instead of MFN2 is required to regulate Opa1-driven mitochondrial fusion [39]. Consida target of miR-125b, we performed luciferase reporter gene experiment and Western blotting, and confirmed that miR-125b could indeed suppress MFN1 levels. Combined with our previous observation that MFN1 levels were repressed in response to cisplatin treatment, miRcess. Our data indicated that miR-125b could regulate mitochondrial division and AKI pathological process via indemonstrated that during cisplatin-induced AKI, miRsions, which attributed to excessive mitochondrial fission and resulted in tubular cell apoptosis. Furthermore, we illustrated the applicability of $\mathrm{miR}-125 \mathrm{~b}$ as a therapeutic

\section{Statement of Ethics}

The experiments conducted in this study received the approval of the Animal Care and Use Committee of Jinling Hospital, Jiangsu, China (2016NZGKJ-043).

\section{Conflict of Interest Statement}

Zhihong Liu is the Editor-in-Chief of the journal Kidney Diseases. The other authors have no conflicts of interest to declare.

\section{Funding Sources}

This work was supported by the Program of Clinical Research Center for Kidney Diseases of Jiangsu Province (YXZXA2016003) and the grants from the National Natural Science Foundation of China (81600559 and 81800629).

\section{Author Contributions}

Y.Z. and Z.L. designed the research; Y.Z., Y.L., M.Z., S.L., and X.Z. performed the experiments; Y.Z. and Y.L. analyzed the data and drafted the manuscript; Z.L. reviewed the literature and revised the manuscript. All authors read and approved the final manuscript.

\section{Data Availability Statement}

All data generated or analyzed during this study are included in this article. ering that MFN1 instead of MFN2 has been predicted as $125 \mathrm{~b}$ inhibition can alleviate cisplatin-induced AKI prohibiting MFN1 expressions. In this study, we $125 \mathrm{~b}$ is upregulated and directly inhibited MFN1 exprestarget in cisplatin nephritis.

References
1 Chacinska A, Koehler CM, Milenkovic D, Lithgow T, Pfanner N. Importing mitochondrial proteins: machineries and mechanisms. Cell. 2009 Aug; 138(4):628-44.

2 Brooks C, Wei Q, Cho SG, Dong Z. Regulation of mitochondrial dynamics in acute kidney injury in cell culture and rodent models. J Clin Invest. 2009 May;119(5):1275-85.

3 Scorrano L, Ashiya M, Buttle K, Weiler S, Oakes SA, Mannella CA, et al. A distinct pathway remodels mitochondrial cristae and mobilizes cytochrome c during apoptosis. Dev Cell. 2002 Jan;2(1):55-67.

4 Whitley BN, Engelhart EA, Hoppins S. Mitochondrial dynamics and their potential as a therapeutic target. Mitochondrion. 2019 Nov; 49:269-83.

5 Emma F, Montini G, Parikh SM, Salviati L. Mitochondrial dysfunction in inherited renal disease and acute kidney injury. Nat Rev Nephrol. 2016 May;12(5):267-80.

6 Lan R, Geng H, Singha PK, Saikumar P, Bottinger EP, Weinberg JM, et al. Mitochondrial pathology and glycolytic shift during proximal tubule atrophy after ischemic AKI. J Am Soc Nephrol. 2016;27(11):3356-67. 
7 Gonsalez SR, Cortês AL, Silva RCD, Lowe J, Prieto MC, Silva Lara LD. Acute kidney injury overview: from basic findings to new prevention and therapy strategies. Pharmacol Ther. 2019 Aug;200:1-12.

8 Linkermann A, Chen G, Dong G, Kunzendorf U, Krautwald S, Dong Z. Regulated cell death in AKI. J Am Soc Nephrol. 2014 Dec;25(12): 2689-701.

$9 \mathrm{Hu}$ Y, Yang C, Amorim T, Maqbool M, Lin J, $\mathrm{Li}$ C, et al. Cisplatin-mediated upregulation of APE2 binding to MYH9 provokes mitochondrial fragmentation and acute kidney injury. Cancer Res. 2021 Feb;81(3):713-23.

10 Ambros V. The functions of animal microRNAs. Nature. 2004 Sep;431(7006):350-5.

11 Wei Q, Bhatt K, He HZ, Mi QS, Haase VH, Dong $Z$. Targeted deletion of dicer from proximal tubules protects against renal ischemiareperfusion injury. J Am Soc Nephrol. 2010 May;21(5):756-61.

12 Wang K, Long B, Jiao JQ, Wang JX, Liu JP, Li $\mathrm{Q}$, et al. MiR-484 regulates mitochondrial network through targeting Fis1. Nat Commun. 2012 Apr;3:781-9.

13 Wei Q, Sun H, Song S, Liu Y, Liu P, Livingston MJ, et al. MicroRNA-668 represses MTP18 to preserve mitochondrial dynamics in ischemic acute kidney injury. J Clin Invest. 2018 Dec; 128(12):5448-64.

14 Le MT, Shyh-Chang N, Khaw SL, Chin L, Teh C, Tay J, et al. Conserved regulation of p53 network dosage by microRNA-125b occurs through evolving miRNA-target gene pairs. PLoS Genet. 2011 Sep;7(9):e1002242-11.

15 Chaudhuri AA, So AY, Mehta A, Minisandram A, Sinha N, Jonsson VD, et al. Oncomir $\mathrm{miR}-125 \mathrm{~b}$ regulates hematopoiesis by targeting the gene Lin28A. Proc Natl Acad Sci USA. 2012 Mar;109(11):4233-8.

16 Giroud M, Pisani DF, Karbiener M, Barquissau V, Ghandour RA, Tews D, et al. miR-125b affects mitochondrial biogenesis and impairs brite adipocyte formation and function. Mol Metab. 2016 Jun;5(8):615-25.

17 Schrepfer E, Scorrano L. Mitofusins, from mitochondria to metabolism. Mol Cell. 2016 Mar;61(5):683-94.
18 Barsoum MJ, Yuan H, Gerencser AA, Liot G, Kushnareva Y, Gräber S, et al. Nitric oxideinduced mitochondrial fission is regulated by dynamin-related GTPases in neurons. EMBO J. 2006 Aug;25(16):3900-11.

19 Li LY, Luo X, Wang X. Endonuclease G is an apoptotic DNase when released from mitochondria. Nature. 2001 Jul;412(6842):95-9.

20 Mishra J, Mori KM Ma Q, Kelly C, Barasch J, Devarajan P. Neutrophil gelatinase-associated lipocalin: a novel early urinary biomarker for cisplatin nephrotoxicity. Am J Nephrol. 2004 May;24(3):307-15.

21 Yuan Y, Wang H, Wu Y, Zhang B, Wang N, Mao H, et al. P53 contributes to cisplatin induced renal oxidative damage via regulating P66shc and MnSOD. Cell Physiol Biochem. 2015 Oct;37(4):1240-56.

22 Zhang C, Zhang W, Chen HM, Liu C, Wu J, Shi S, et al. Plasma microRNA-186 and proteinuria in focal segmental glomerulosclerosis. Am J Kidney Dis. 2015 Feb;65(2):223-32.

23 Duroux-richard I, Roubert C, Ammari M, PrésumeyGr JJR, Grün JR, Häupl T, et al. miR-125b controls monocyte adaptation to inflammation through mitochondrial metabolism and dynamics. Blood. 2016 Dec; 128(26):3125-36

24 Faul C, Donnelly M, Merscher-Gomez S, Chang YH, Franz S, Delfgaauw J, et al. The actin cytoskeleton of kidney podocytes is a direct target of the antiproteinuric effect of cyclosporine A. Nat Med. 2008 Sep;14(9):931-

25 Bhargava P, Schnellmann RG. Mitochondrial energetics in the kidney. Nat Rev Nephrol. 2017 Oct;13(10):629-46.

26 Galvan DL, Green NH, Danesh FR. The hallmarks of mitochondrial dysfunction in chronic kidney disease. Kidney Int. 2017 Nov; 92(5):1051-7.

27 Tábara LC, Poveda J, Martin-Cleary C, Selgas R, Ortiz A, Sanchez-Niño MD. Mitochondria-targeted therapies for acute kidney injury. Expert Rev Mol Med. 2014 Aug;16:e13-25.

28 Szeto HH, Liu S, Soong Y, Seshan SV, CohenGould L, Manichev V, et al. Mitochondria protection after acute ischemia prevents prolonged upregulation of IL- $1 \beta$ and IL-18 and arrests CKD. J Am Soc Nephrol. 2017 May; 28(5):1437-49.
29 Liu Z, Li H, Su J, Xu S, Zhu F, Ai J, et al. Numb depletion promotes drp1-mediated mitochondrial fission and exacerbates mitochondrial fragmentation and dysfunction in acute kidney injury. Antioxid Redox Signal. 2019 May;30(15):1797-816.

30 Baker MA, Davis SJ, Liu P, Pan X, Williams AM, Iczkowski KA, et al. Tissue-specific MicroRNA expression patterns in four types of kidney disease. J Am Soc Nephrol. 2017 Oct; 28(10):2985-92.

31 Sun YM, Lin KY, Chen YQ. Diverse functions of miR-125 family in different cell contexts. J Hematol Oncol. 2013 Jan;6(1):6.

32 Zheng Z, Qu JQ, Yi HM, Ye X, Huang W, Xiao $\mathrm{T}$, et al. MiR-125b regulates proliferation and apoptosis of nasopharyngeal carcinoma by targeting A20/NF- $\mathrm{kB}$ signaling pathway. Cell Death Dis. 2017 Jun;8(6):e2855.

33 Gong J, Zhang JP, Li B, Zeng C, You K, Chen MX, et al. MicroRNA-125b promotes apoptosis by regulating the expression of $\mathrm{Mcl}-1, \mathrm{Bcl}-$ $\mathrm{w}$ and IL-6R. Oncogene. 2013 Jun;32(25): 3071-9.

34 Wang JX, Jiao JQ, Li Q, Long B, Wang K, Liu JP, et al. MiR-499 regulates mitochondrial dynamics by targeting calcineurin and dynamin-related protein-1. Nat Med. 2011 Jan; 17(1):71-8.

35 Purohit PK, Edwards R, Tokatlidis K, Saini N. MiR-195 regulates mitochondrial function by targeting mitofusin-2 in breast cancer cells. RNA Biol. 2019 Jul;16(7):918-29.

36 Liu T, Wang B, Li G, Dong X, Yu G, Qian Q, et al. Disruption of microRNA-214 during general anaesthesia prevents brain injury and maintains mitochondrial fusion by promoting Mfn2 interaction with Pkm2. J Cell Mol Med. 2020 Dec;24(23):13589-99.

37 Ishihara N, Eura Y, Mihara K. Mitofusin 1 and 2 play distinct roles in mitochondrial fusion reactions via GTPase activity. J Cell Sci. 2004 Dec;117(26):6535-46.

38 Cipolat S, Martins de Brito O, Dal Zilio B, Scorrano L. OPA1 requires mitofusin 1 to promote mitochondrial fusion. Proc Natl Acad Sci USA. 2004 Nov;101(45):15927-32.

39 Li J, Li Y, Jiao J, Wang J, Li Y, Qin D, et al Mitofusin 1 is negatively regulated by microRNA 140 in cardiomyocyte apoptosis. Mol Cell Biol. 2014 May;34(10):1788-99. 